\title{
Perfil dos Pacientes Atendidos no Ambulatório de Cardiologia da Região Sul do
} Tocantins no Ano de 2015

\section{Profile of Patients Attended in the Cardiology Ambulatory of the South Region of Tocantins in the Year 2015}

Maira Moura Dantas ${ }^{1}$, Alda Lúcia Nunes Solá2, Danyelle Amorim de Lima Pires², João Batista Pereira da Silva², Milena Aparecida Carneiro dos Reis ${ }^{2}$, Marcos Renato Herrera $^{3}$, Sávia Denise Silva Carlotto Herrera ${ }^{4}$

\section{RESUMO}

As doenças do aparelho circulatório, por sua importância e magnitude, constituem-se em um dos mais importantes problemas de saúde da atualidade. Elas correspondem à primeira causa de óbito em todas as regiões do país. O objetivo desse estudo foi avaliar o perfil dos pacientes atendidos no ambulatório de cardiologia do Universidade de Gurupi no ano de 2015. Para tanto, após a aprovação do Comitê de Ética em Pesquisa com Seres Humanos, foram avaliados os prontuários, que apresentavam informações completas referentes as variáveis: Hipertensão (HAS), Acidente Vascular Cerebral, sexo, idade, tabagismo e etilismo. Do total de 86 pacientes aleatórios, observou-se diferença significativa na incidência de pacientes com HAS e tabagismo e entre HAS e etilismo. Em relação ao acidente vascular cerebral, 5,9\% destes pacientes sofriam ou já sofreram com a doença. $\mathrm{Na}$ avaliação da faixa etária, o etilismo se comporta de forma inconstante, sendo prevalente na meia idade e diminui sua incidência a partir da faixa entre 61-90 anos. Contatou-se que, no ano de 2015 , mais de $50 \%$ dos pacientes atendidos no ambulatório de cardiologia de Gurupi - TO são hipertensos, e que o perfil desses pacientes, de forma geral, segue o padrão nacional.

Palavras-chave: Doenças do aparelho circulatório; Cardiologia; Epidemiologia; Acidente vascular cerebral; Tabagismo.

\section{ABSTRACT}

The diseases of the circulatory system, because of their importance and magnitude, constitute one of the most important health problems of the present time. They are the number one cause of death in all regions of the country. The purpose of this study was to evaluate the profile of the patients treated at the cardiology outpatient clinic of the University of Gurupi in the year 2015. For this purpose, after the approval of the Ethics Committee in Research with Human Beings, the medical records were evaluated, which presented full information regarding the variables: Hypertension (SAH), cerebrovascular accident, sex, age, smoking, alcohol consumption. From the total of 86 random patients, a significant difference was observed in the incidence of SAH and smoking patients and between SAH and alcoholism. In relation to cerebrovascular accident, 5.9\% of these patients suffered or already suffered from the disease. In the evaluation of the age group, the alcoholism behaves inconsistently, being prevalent in middle age and decreasing its incidence from the range between $61-90$ years. In the year 2015 , more than $50 \%$ of the patients treated at the Gurupi-TO cardiology outpatient clinic were hypertensive, and that the profile of these patients generally follows the national standard.

Keywords: Diseases of the circulatory system; Cardiology; Epidemiology; Cerebrovascular accident; Smoking.

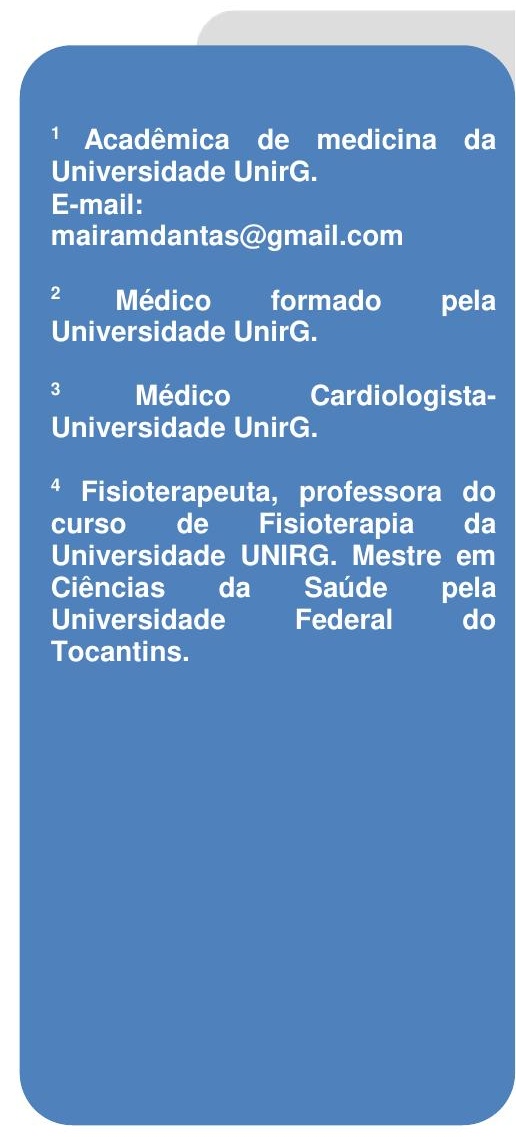




\section{INTRODUÇÄO}

A prevalência da doença coronariana vem aumentando progressivamente em todo o mundo, com maior índice de mortalidade no Brasil e nos países desenvolvidos, em ambos os sexos, entre 50 e 64 anos. Estimativas apontam que em 2020 esse número deve elevarse, permanecendo como a doença de maior mortalidade e incapacitação, trazendo consigo prejuízos e gastos públicos alarmantes. Apesar da alta incidência e da gravidade, a doença coronariana pode ser evitada com medidas de controle dos fatores de risco (NOGUEIRA et Al., 2016).

A hipertensão é caracterizada por elevação sustentada dos níveis pressóricos $\geq 140$ e/ou $90 \mathrm{mmHg}$. Frequentemente se associa a distúrbios metabólicos, alterações funcionais e/ou estruturais de órgãos-alvo, sendo agravada pela presença de outros fatores de risco (FR), como dislipidemia, obesidade abdominal, intolerância à glicose e diabetes melito (DM). Mantém associação independente com eventos como morte súbita, acidente vascular encefálico (AVE), infarto agudo do miocárdio (IAM), insuficiência cardíaca (IC), doença arterial periférica (DAP) e doença renal crônica (DRC), fatal e não fatal (SBC, 2016).

No Brasil, estudos que envolvem análise da prevalência da HAS indicam que $65 \%$ dos hipertensos são idosos e em sua maioria mulheres acima dos 75 anos, com mais prevalência em raça negra em ambos os sexos. A doença cerebrovascular, como complicação da HAS, se destaca, sendo responsável por $65 \%$ das mortes por AVC. Ao investigar a presença de fatores de risco cardiovascular em pacientes diagnosticados com AVC, foram detectados hipertensão em $68,8 \%$; sedentarismo em $65,6 \%$; diabetes mellitus tipo II em 20,3\%; tabagismo em 9,4\%, hipercolesterolemia em 9,4\% e sedentarismo associado à hipertensão em 75,7\% (RODRIGUES et al., 2015).

O controle da hipertensão está intimamente relacionado às mudanças de hábitos de vida, como: alimentação adequada, prática regular de exercícios físicos e abandono do tabagismo. Com isso, destaca-se que, para o sucesso do controle da hipertensão arterial sistêmica, é necessária a prática do autocuidado pelo paciente. Este é definido como a realização de atividades que os indivíduos desempenham em seu benefício para manter a vida, a saúde e o bem-estar. Quando o autocuidado é efetivamente realizado, ajuda a manter a integridade estrutural e funcional, contribuindo para o desenvolvimento humano. Entretanto, quando esse autocuidado não é realizado pelo paciente com hipertensão, surgem os déficits de autocuidado, sendo essencial, nessa situação, a inserção do 
profissional de saúde para atuar na sensibilização dos pacientes na adesão à prática do autocuidado, visando prevenir complicações e promover a saúde (MENDES et al., 2016).

A prática regular de atividades físicas é parte primordial das condutas não medicamentosas de prevenção e tratamento da HAS. Segundo diretrizes nacionais e internacionais, todos os pacientes hipertensos devem fazer exercícios aeróbios complementados pelos resistidos, como forma isolada ou complementar ao tratamento medicamentoso (SBC, 2016). A HAS é uma doença que atinge todas as idades, mas principalmente pessoas acima dos 35 anos e idosos, sendo imprescindível, para que esse grupo de pessoas tenha um futuro com mais qualidade de vida, uma mudança comportamental em relação do que é observado hoje, utilizando a prática de exercícios físicos na prevenção dessas doenças (MATAVELLI et al., 2014).

A idade se constitui como um fator de risco para hipertensão, pois ocorrem alterações na musculatura lisa e no tecido conjuntivo dos vasos sanguíneos devido ao envelhecimento. Um estudo feito em Pelotas-RS com 1.968 pessoas comprova esta afirmação uma vez que mais da metade dos idosos com idades entre 60 e 69 anos são hipertensos, sendo que estes correspondem a $11,6 \%$ do total de participantes (MACHADO et al., 2012).

Segundo Jardim (2014) o número estimado de pessoas que morrerão por Doenças cardiovasculares (DCV), principalmente doença cardíaca (DC) e acidente vascular cerebral (AVC), está estimado em 23,3 milhões em 2030, permanecendo como a principal causa de morte no mundo. A Organização Mundial da Saúde - OMS (2014) estima que três quartos da mortalidade cardiovascular pode ser diminuída com adequadas mudanças no estilo de vida, e esse é o grande desafio das diversas diretrizes existentes em prevenção cardiovascular.

O controle da HAS constitui uma das áreas estratégicas da Atenção Primária à Saúde (APS). As estratégias educativas constituem um importante instrumento para estimular mudanças no estilo de vida e reduzir os fatores de risco cardiovascular. Estudos têm analisado a importância, a efetividade e as limitações dessas estratégias no tratamento da HAS. Como resultados têm sido observados a redução da pressão arterial, a diminuição do peso corporal e da circunferência da cintura, melhora do perfil lipídico e da glicose sanguínea, mudanças favoráveis no consumo alimentar habitual e aumento do conhecimento sobre o processo saúde-doença-cuidado (MACHADO et al., 2014).

Assim, este estudo teve por objetivo avaliar o perfil dos pacientes atendidos no ambulatório de cardiologia do Universidade de Gurupi no ano de 2015, uma vez que produz dados para aprimoramento das ações educativas em saúde. 


\section{MATERIAIS E METODOS}

Foi realizado um estudo de campo analítico, evidenciando o perfil epidemiológico dos pacientes que frequentaram o ambulatório de cardiologia do Universidade de Gurupi, região sul do Tocantins. Foram selecionados os prontuários que apresentavam informações completas referentes as variáveis estudadas, Hipertensão (HAS), Acidente Vascular Cerebral (AVC), sexo, idade, tabagismo e etilismo no ano de 2015. A coleta foi realizada na sala de prontuários do ambulatório de saúde comunitária do Universidade de Gurupi, que foram avaliados após a autorização do Comitê de Ética em Pesquisa com CAAE 61991216.4.0000.5518, sendo usados tendo atenção à resolução do CNS n 466/2012.

Após a análise dos prontuários disponibilizados na sala de arquivos do ambulatório de cardiologia averiguou-se que 133 dos documentos correspondiam a pacientes atendidos no ano de 2015 , destes foram estudados apenas 86 pacientes $(n=86)$ com diferentes faixas etárias (entre 21 e 90 anos), sexo (57 mulheres e 29 homens), incidência de HAS, e hábitos como tabagismo e etilismo, por apresentarem dados mais completos que possibilitavam o estudo. Excluiu-se da coleta e análise os prontuários com informações incompletas. $O$ baixo número de prontuários do ano citado deve-se a não informatização do sistema, o que gera desorganização do armazenamento e até mesmo a perda de prontuários.

Todos os dados foram inseridos em planilhas EXCEL 2013, reagrupados no perfil de análise, pacientes com HAS, com hábitos de etilismo e com hábitos de tabagismo de acordo com sua faixa etária e sexo e pacientes com AVC. A análise estatística foi feita para os grupos, HAS x Tabagismo, HAS x Etilismo, HAS x Idade, HAS x Sexo e AVC x Idade usando o programa ASSISTAT, versão 7.7 beta (pt), sendo que tais grupos passaram por adequações a partir de testes normalidade de Shapiro Wilk a um nível de $5 \%$ de significância $(p<0,05)$ para verificar a distribuição normal dos dados coletados, em caso de dados sem normalidade, foi utilizada a raiz quadrada de cada média. Nas variáveis descritas, para análise dos grupos, utilizou-se teste de Friedman, afim de verificar se existe diferença significativa das médias dentro dos grupos.

Com a diversidade do grupo idade, os mesmos foram divididos em faixas correspondentes a determinado grupo etário, afim de obter uma melhor análise conjunta deste parâmetro. 


\section{RESULTADOS}

Do total de 86 pacientes avaliados ( $n=86), 66,27 \%$ eram mulheres $(n=57), 68,60 \%$ eram hipertensos ( $n=59), 22,09 \%$ tabagistas $(n=19), 18,60 \%$ etilistas $(n=16)$ e $5,81 \%$ tiveram AVC $(n=5)$.

A incidência de pacientes com quadro de AVC, acometido alguma vez na vida, possui diferença significativa em relação à população total avaliada ( $p=0,0009)$, com um total de 5 pacientes vítimas deste quadro. Implicando que de uma população de 86 pacientes, 5,81\% destes já tiveram AVC. O mesmo ocorre ao avaliar a incidência de pacientes com HAS e tabagismo ( $p=0,0001)$ e entre HAS e etilismo $(p=0,0001)$, sendo que a quantidade de pacientes com HAS é 59, com tabagismo 19 e etilismo 16.

Com os resultados apresentados na figura 1, demonstra-se que não há diferença significativa, estatisticamente, quanto ao etilismo em todas as faixas etárias, e seu maior valor, $n=4$, pode ser verificado em duas faixas etárias, 41-50 anos e 71-80 anos. É possível verificar que existe diferença significativa parcial no hábito de tabagismo apenas nas faixas etárias de 41-50 e 71-80 anos, com $n=6$ e $n=7$, respectivamente; em outras faixas etárias não há diferença significativa quanto ao tabagismo. Em relação a incidência de HAS por faixa etária, começa a existir diferença significativa parcial a partir da faixa entre 31-40 anos, com um valor de $n=6$, tal diferença significativa é total a partir da faixa entre 41-50 e 51-60 anos, com valor $n=10$, o maior valor da incidência de HAS é atingido na faixa etária entre 71-80 anos, cuja média atinge o valor de $n=16$; em contrapartida, há uma diminuição significativa desta incidência na faixa etária entre 81-90 anos, com um valor médio de $n=3$. Nas faixas etárias entre 0 e 30 anos pode-se perceber a não incidência de HAS, tabagismo e etilismo, colaborando com a premissa de que a HAS é um fator temporal e que, de alguma forma, consequente de certos hábitos de vida. $\mathrm{Na}$ avaliação da faixa etária, o etilismo se comporta de forma inconstante, sendo prevalente na meia idade, de 41-50 anos, e diminui sua incidência a partir da faixa entre 81-90 anos. 


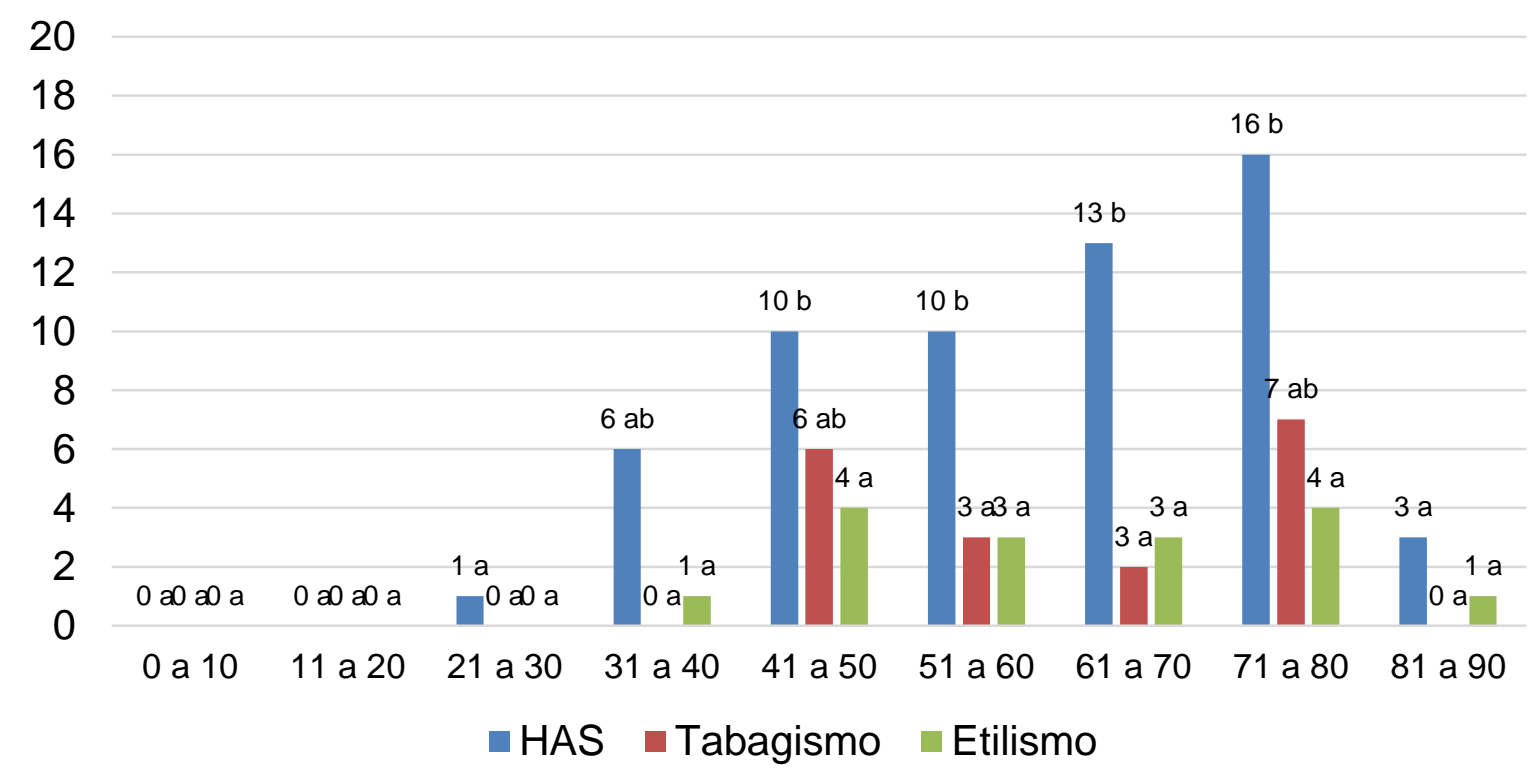

Figura1 - Gráfico quantitativo médio de pacientes com HAS, tabagismo e etilismo de acordo com a faixa etária durante $\mathrm{o}$ ano de 2015.

Quando se relaciona HAS e hábitos de tabagismo e etilismo com o fator sexo, é possível verificar, e melhor ilustrado pela figura 2, que do total de mulheres $(n=57)$, aproximadamente $70 \%$ são acometidas por HAS e do total de homens ( $n=29$ ), aproximadamente $65,5 \%$ são hipertensos. Ao analisar o total de pacientes hipertensos $(n=59), 67,7 \%$ são do sexo feminino.

Observando-se o fator tabagismo, não existe uma diferença significativa entre os sexos, onde aproximadamente $17,54 \%$ das mulheres tem hábitos de tabagismo $(n=10)$ e aproximadamente $31,03 \%$ dos homens possuem o mesmo hábito $(n=9)$, seguindo a relação de HAS com o tabagismo, podemos prever que terá o mesmo comportamento quando relacionados ao fator sexo.

Em relação ao etilismo também não é existente uma diferença significativa entre homens e mulheres; onde do total de mulheres, $28,07 \%$ delas possuem hábitos de etilismo, enquanto $34,48 \%$ do total de homens praticam do mesmo hábito. Dessa forma, a mesma premissa de que HAS e etilismo possuem um perfil semelhante é válida, uma vez que tal hábito de etilismo pode ser diretamente relacionado ao acometimento de HAS. 


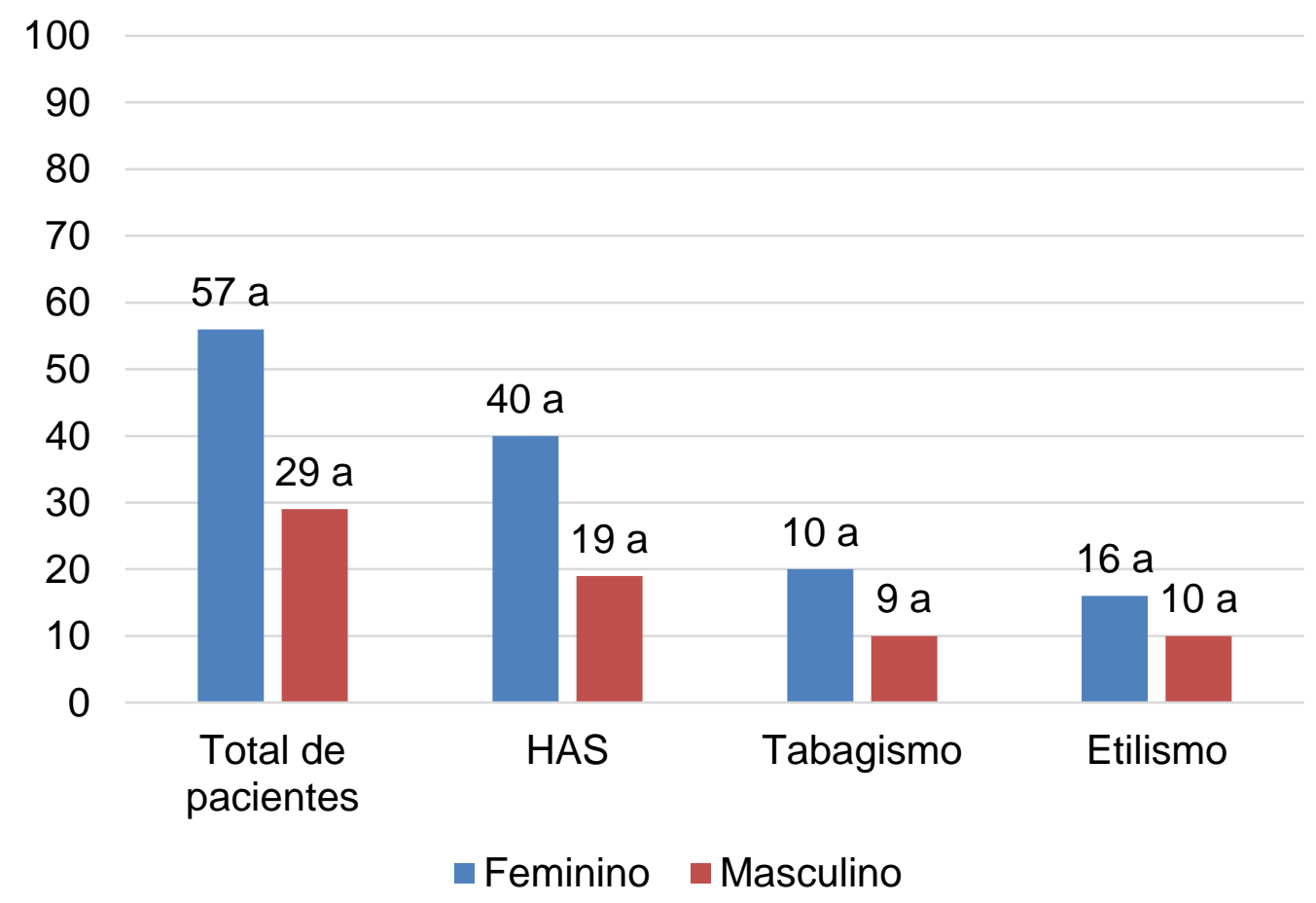

Figura 2 - Gráfico quantitativo médio de pacientes com HAS, tabagismo e etilismo de acordo com o sexo durante $o$ ano de 2015.

A incidência de AVC de acordo com a faixa etária, não demonstrou diferença significativa entre todas as faixas analisadas. Ainda assim, observa-se um início de incidência do AVC a partir de 61-70 anos, aumentando tal incidência na faixa entre 71-80 anos e prorrogando-se, em menor valor, na faixa entre 81-90 anos, corroborando com o fato de que a idade associada a fatores de riscos é crucial para este perfil de AVC, mais incidente em idosos.

\section{DISCUSSÁO}

Durante o XXIII Congresso Brasileiro de Hipertensão, organizado pela Sociedade Brasileira de Hipertensão, demonstrou-se a preocupação com o crescimento da hipertensão arterial no Brasil e no mundo. Segundo o estudo de 2015, Heart Disease and Stroke Statistics (Estatísticas sobre doenças cardíacas e infartos), da American Heart Association, durante a última década pesquisada, de 2001 a 2011, a taxa de morte por hipertensão, em mais de 190 países pesquisados, aumentou 13,2\%. (MOZAFFARIAN et al., 2014). A preocupação é grande e torna-se ainda maior, pois apesar da redução de $30,8 \%$ nas taxas de morte por doença cardiovascular de 2001 a 2011, percebe-se que a hipertensão arterial se encontra em uma tendência crescente e tende a acometer a população de forma cada vez mais intensa, devido aos inúmeros fatores de risco e a maior 
expectativa de vida. Corroborando com este estudo que mostrou que $68,6 \%$ dos pacientes são hipertensos e dentre os hipertensos $54,23 \%$ encontram-se na faixa etária de 61 a 90 anos.

Percebe-se claramente que o surgimento da HAS é mais significativo em pacientes com idades mais avançadas. O envelhecimento está entre os principais fatores de risco para doença cardiovascular. Visto que há vários mecanismos fisiológicos associados ao avançar da idade e que favorecem a elevação dos níveis pressóricos: aumento da rigidez arterial e perda da elasticidade, minimização da sensibilidade dos barorreceptores, aumento da atividade do sistema nervoso simpático e a minimização da capacidade renal de excreção de sódio (BORTOLOTTO, 2012). Todos esses fatores contribuem para um denominador comum que é a perda da qualidade da função cardíaca.

A HAS é a morbidade mais prevalente entre idosos, superior a 50\%, sendo uma das principais causas de óbito (TAVARES et al., 2014). E na região estudada a situação foi a mesma, com prevalência entre 61 e 90 anos. Na grande maioria das vezes, a causa de HAS é desconhecida. O componente genético é importante causa para uma pequena parcela dos hipertensos e a interação da genética com múltiplos fatores fisiológicos associados a fatores comportamentais são responsáveis pela maioria dos casos (LOPES, 2014). A obesidade, sedentarismo, tabagismo, hipertensão, dislipidemia e consumo excessivo de álcool são as principais razões para a ocorrência de obstrução arterial e consequentemente gerar uma influência significativa no fluxo sanguíneo (CARVALHO et al., 2015).

A doença arterial coronariana (DAC) é um quadro clínico no qual o suprimento de sangue para o músculo cardíaco é bloqueado parcial ou completamente, a determinação dos fatores de risco para DACs ainda gera muitos investimentos em pesquisas devido à alta morbimortalidade da população, mesmo que muitos dos fatores já estejam bem estabelecidos. Pois esses fatores estão presentes diversas vezes em indivíduos aparentemente hígidos. A HAS é a condição mais prevalente, sendo o AVC a sua complicação de maior gravidade. A associação de HAS com a maioria das DACs já está bem estabelecida (GUS e COLS, 2015).

Os dados apresentados reforçam a importância do AVC como comorbidade associada à HAS. Dentro do contexto da saúde pública, o AVC deve ser destacado como importante causa de morte no Brasil (BRITO et al., 2011). No presente estudo a incidência de pacientes com quadro de $A V C$, foi significativa em relação à população total avaliada, corroborando 
com o fato de que a idade associada a fatores de riscos é crucial para este perfil patológico, mais incidente em idosos, como já demonstrado em outros estudos.

É preciso salientar que existe um maior número de pacientes do sexo feminino pelo fato de que as mulheres são mais ativas na procura de tratamentos em postos de saúde do que os homens. Entre os hábitos negativos abordados nessa pesquisa, destacam-se 0 tabagismo e o etilismo como os fatores que aceleram o desenvolvimento da HAS. O tabagismo é a principal causa de morte passível de prevenção. O cigarro duplica o risco de doença arterial coronariana. Tanto a nicotina quanto álcool têm efeitos de aumentar a pressão arterial. A nicotina causa a liberação de catecolaminas, que além de aumentar a pressão arterial, aumenta a frequência cardíaca e a resistência periférica. Os estudos apontam uma correlação positiva do álcool com a HAS, mas as evidências não são consistentes em relação aos riscos cardiovasculares que o etanol exerce em indivíduos hipertensos (SOUZA, 2014).

De acordo com a Organização Mundial da Saúde, a hipertensão é responsável por 9,4 milhões de mortes no mundo, atingindo $30 \%$ da população adulta brasileira, chegando a mais de $50 \%$ na terceira idade e está presente em $5 \%$ das crianças e adolescentes no Brasil. Portanto, a melhor conduta é a prevenção, e é essa a importância deste estudo, mostrando a realidade de uma cidade do Sul do Tocantins, para assim reforçar a necessidade de medidas preventivas e educativas. Segundo a Sociedade Brasileira de Cardiologia, homens sem histórico familiar de doenças cardiovasculares podem visitar o médico a cada cinco anos até completar 40 anos e uma vez por ano a partir dessa idade para assim, ser identificado os fatores de risco e uma melhor intervenção. É importante salientar que, o comportamento preventivo ajuda, porém não afasta as chances de o problema aparecer (OMS, 2014).

\section{CONSIDERACOOES FINAIS}

Conclui-se que $66,27 \%$ dos pacientes atendidos no ambulatório de cardiologia do Universidade de Gurupi eram do sexo feminino, $68,60 \%$ eram hipertensos e que a idade mais acometida pela HAS está entre 71 e 80 anos. Observou-se que a quantidade de pacientes com HAS foi cerca de três vezes maior que a quantidade de pacientes com tabagismo e etilismo e que $5,81 \%$ desses pacientes sofrem ou já sofreram com AVC, sobretudo em idades mais avançadas (71 -80 anos). 
A estatística utilizada demonstrou diferença significativa entre HAS e tabagismo e HAS e etilismo, corroborando com a importância dos fatores de risco modificáveis para o desenvolvimento de doenças cardiovasculares. Também foi significante o número de AVC entre os pacientes avaliados. A prevalência da HAS foi maior no sexo feminino, tabagista e com idade mais avançada. Quanto aos fatores de risco não modificáveis evidenciou-se que o avançar da idade tem significância tanto para HAS como para AVC, sendo assim, a informação e o incentivo para uma melhor qualidade de vida e, portanto, um envelhecer mais saudável é crucial para diminuição dessa estatística entre os idosos.

Diante do perfil epidemiológico traçado é possível direcionar as políticas de assistência básica. Com base nas evidências, a educação em saúde é uma ferramenta fundamental na prevenção da HAS, uma vez que por meio dela pode-se promover o conhecimento sobre a doença e suas consequências, além de alertar o paciente sobre os fatores de risco.

\section{REFERÉNCIAS}

BORTOLOTTO, L. A. Mecanismos Fisiopatológicos da Hipertensão no Idoso. Rev. Bras. Hipertensão. vol. 19(3):61-64, 2012.

BRITO, E. S; PANTAROTTO, R. F. R; COSTA, L. R. L. G. A Hipertensão Arterial Sistêmica Como Fator De Risco Ao AVE. J Health Sci. Inst. 2011;29(4):265-8.

CARVALHO, C. A. et al. Associação entre Fatores de Risco Cardiovascular e Indicadores Antropométricos de Obesidade em Universitários de São Luís, Maranhão, Brasil. Ciência \& Saúde Coletiva, 20(2):479-490, 2015DOI: 10.1590/141381232015202.02342014 .

GUS E COLS. Fatores de Risco para DAC: 2002-2014. Arq. Bras. Cardiologia. 2015; 105(6):573-579.

LOPES, H. F. Genética e Hipertensão Arterial. Rev. Bras. Hipertensão. vol. 21(2):87-91, 2014.

MACHADO, J. C. et al. Análise de Três Estratégias de Educação em Saúde para Portadores de Hipertensão Arterial. Ciência \& Saúde Coletiva. Rio de Janeiro, n.2, v.21, 20 dez.2014, p.611-628.

MACHADO, M. C. et al. Concepções dos Hipertensos Sobre os Fatores de Risco para a Doença. Ciência \& Saúde Coletiva. Rio de Janeiro, n.5, v.17, maio.2012, p.1357-1363.

MATAVELLI, I. S. et al. Hipertensão Arterial Sistêmica e a Prática Regular de Exercícios Físicos como Forma de Controle: Revisão de Literatura. Revista Brasileira de Ciências da Saúde. São Paulo, n. 4, v.18, abril.2014, p. 359-366.

MENDES, C. R. S. et al. Prática de autocuidado de pacientes com hipertensão arterial na atenção primária de saúde. Rev Rene. 2016 jan-fev; 17(1):52-9. 
MOZAFFARIAN, D. et al. Heart Disease and Stroke Statistics-2015 Update. Circulation. 2015; 131: e29-e322, originally published December 17, 2014.

NOGUEIRA, J. S. et al. Fatores de Risco Cardiovascular e Doença Coronariana: uma análise em pacientes revascularizados. Rev. Pesq. Saúde, 17(1):37-41, jan-abr, 2016.

ORGANIZAÇÃO MUNDIAL DE SAÚDE. OMS. Doenças Cardiovasculares Causam Quase $30 \%$ das Mortes no País. Portal Brasil, 2014. Disponível em: <http://www.brasil.gov.br/saude/2011/09/doencas-cardiovasculares-causam-quase-30das-mortes-no-pais> Acesso em 30 de janeiro de 2017.

RODRIGUES, E. S. R. et al. Perfil dos Fatores de Risco Cardiovascular em Pacientes Hipertensos. 10.18605/2175-7275/cereus.v7n2p88-104, maio-agosto/2015, UnirG, Gurupi, TO, Brasil.

SOCIEDADE BRASILEIRA DE CARDIOLOGIA. SBC. $7^{\text {a }}$ Diretriz Brasileira De Hipertensão arterial. Disponível em: <http://publicacoes.cardiol.br/consenso/2010/Diretriz_hipertensao_associados>Acesso em 23 de novembro de 2016.

SOUZA, D. S. M. Álcool e Hipertensão. Aspectos Epidemiológicos, Fisiopatológicos e Clínicos. Rev. Bras. Hipertensão. vol. 21(2):83-86, 2014.

TAVARES, D. M. S. et al. Quality of Life and Accession to the Pharmacological Treatment Among Elderly Hypertensive. Rev. Bras. Enfermagem [Internet]. 2015; 68(6):122-9. DOI: http://dx.doi.org/10.1590/0034-7167.2016690118i. 